\title{
Probing the Nature of Cygnus X-3's Major Radio Flares with INTEGRAL
}

\author{
Michael L. McCollough* \\ $C f A / C X C / S A O$ \\ E-mail: mmccollough@head.cfa.harvard.edu \\ K. I. I. Koljonen \\ CfA/SAO/Univ. of Helsinki \\ E-mail: karri.koljonen@gmail.com \\ D. C. Hannikainen \\ Metsähovi Radio Observatory \\ E-mail: diana@kurp.hut.fi
}

\begin{abstract}
Cygnus X-3 is a well known microquasar which shows state changes, strong radio emission, hard X-ray/soft X-ray/radio correlations, and relativistic jets. In 2006 after a period of almost four years of quiescence Cygnus X-3 transitioned into an active state which included extended quenched emission and several major radio flares $(\sim 14 \mathrm{Jy})$. During this activity a major international observing campaign was undertaken. This included observations on four different spacecraft (INTEGRAL, Chandra, Swift, and RXTE), three different radio observatories (Ryle, RATAN-600, and VLA), and one ground based IR observatory (PAIRITEL).

During this campaign INTEGRAL Target of Opportunity Observations (TOOs) were performed during and following two major radio flares (13.8 Jy at $15 \mathrm{GHz}$ and $13.2 \mathrm{Jy}$ at $2.3 \mathrm{GHz}$ respectively). In this paper we will discuss the hard $\mathrm{X}$-ray $/ \gamma$-ray spectrum of Cygnus $\mathrm{X}-3$ during the major radio flares, show how it evolves, and present Spectral Energy Distributions (SEDs) of the flares. We will also present the results of our fits and discuss the ramifications of our findings.
\end{abstract}

7th INTEGRAL Workshop

September 8-11 2008

Copenhagen, Denmark

\footnotetext{
* Speaker.
} 


\section{Introduction}

Cygnus $\mathrm{X}-3$ is a unique $\mathrm{X}$-ray binary (XRB). Its $\mathrm{X}$-ray emission shows a very strong 4.8 hour orbital modulation which is typical for a low-mass $\mathrm{X}$-ray binary. But its mass-donating companion is a Wolf-Rayet star [1] which makes it a high-mass X-ray binary. Also unlike most other X-ray binaries Cygnus X-3 is relatively bright in the radio virtually all of the time (the exceptions being the quenched states). In addition, Cygnus X-3 undergoes giant radio outbursts and there is strong evidence of jet-like structures moving away from Cygnus X-3 at 0.3-0.9c [2, 边, 母].

Cygnus X-3 has been noted to experience low/hard and high/soft states in the soft X-ray (SXR). Hard X-ray (HXR) and radio studies [5, 6] have shown there to be at least four X-ray/radio states (quiescence, minor flaring, quenched, and major flares). A more recent study of the X-ray and radio behavior [7] have shown evidence for six states (see Section 3). In all states the X-ray spectrum is best represented by a dominant hybrid plasma Comptonization component, a thermal disk component, and several strong emission lines. Also various absorption and reflection components are necessary to understand the system.

\section{Relationship Between the Hard X-Ray and the Radio}

In several studies [8, 9, 10, 11, 6, 12] the 20-100 keV HXR emission, detected from Cygnus $\mathrm{X}-3$ by $C G R O / \mathrm{BATSE}$, was compared with observations in the radio [Green Bank Interferometer (GBI) and Ryle Telescope] and the SXR (RXTE/ASM). The following discoveries hold over all of the overlaps between the data sets: (a) During times of moderate radio brightness ( $\sim 100 \mathrm{mJy})$, and low variability the HXR flux anti-correlates with the radio. It is during this time that the HXR reaches its highest level. (b) During periods of flaring activity in the radio the HXR flux switches from an anti-correlation to a correlation with the radio. In particular, for major radio flares and the quenched radio emission (very low radio fluxes of 10-20 mJy) which precedes them the correlation is strong. (c) The HXR flux has been shown to anti-correlate with the SXR. This occurs in both the low/hard and high/soft X-ray states. (d) the results that the flaring periods in the radio occur during the high/soft X-ray states [13] were confirmed. (e) It was been found from the CGRO/BATSE and CGRO/OSSE data that the spectrum of Cygnus X-3 (in the 20-100 keV band) becomes harder during times of radio flaring [11]. Examples of the various HXR/Radio correlations can be seen in Fig. 1a.

In February 1997 a large radio flare ( 10 Jy) was observed by GBI and Ryle after a period of quenched emission. This flare was detected by CGRO/BATSE in the HXR and showed a strong correlation with the radio. The flare in the HXR was preceded by several days of very low HXR flux (below the CGRO/BATSE one day detection limit). The flare triggered a VLBA observation to obtain high resolution radio images of Cygnus X-3 during the major flare. The resulting VLBA observations show an expanding one-sided jet [ [ and an inclination of $\lesssim 14$ degrees to our line of sight. It appears that Cygnus X-3 is, in some respects, similar to the superluminal sources GROJ1655-40 and GRS1915+105. Cygnus X-3 in fact may represent an example of a galactic microblazar. 

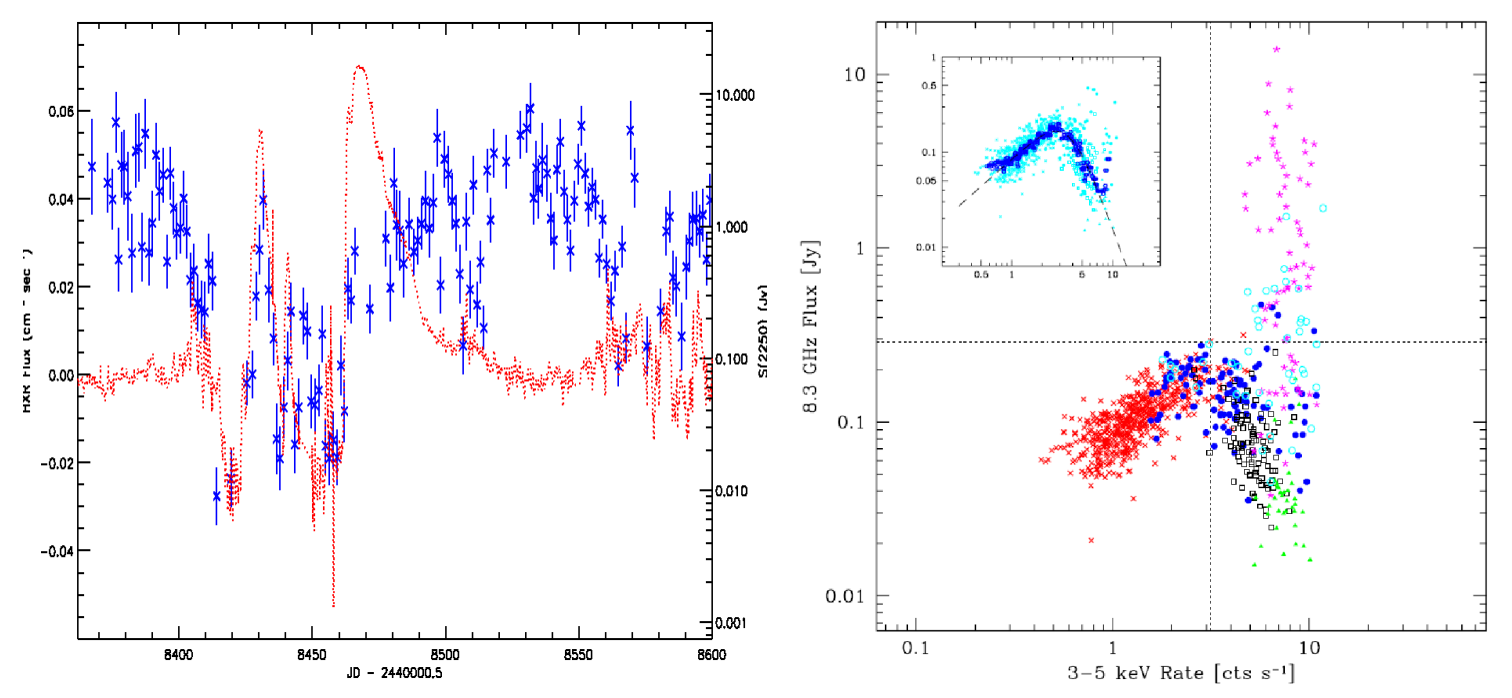

Figure 1: left (a): A plot of the CGRO/BATSE 20-100 keV flux of Cygnus X-3 (blue) for the first 240 days of the CGRO mission. Overlayed on a log scale is the $2.25 \mathrm{GHz}$ flux (red dotted line) measured by the GBI during the same time interval [6]. right (b): Radio vs. X-ray plot from [7] with different states: quiescent (red), minor flaring (blue), suppressed (black), quenched (green), major flaring (magenta), and post-flare (cyan). The insert highlights the transition point mentioned in the text.

\section{X-Ray/Radio States of Cygnus X-3}

In a recent study [7] a revised set of X-ray/radio states to describe the the behavior of Cygnus $\mathrm{X}-3$ was presented. These are based on flux levels (in the radio and X-ray) and variability in these bands. The key transitional flux level was $\sim 3 \mathrm{cts} \mathrm{s}^{-1}$ in the $3-5 \mathrm{keV}$ band of the RXTE/ASM and $\sim 0.25 \mathrm{Jy}$ in the $8.5 \mathrm{GHz}$ GBI radio band. Fig. $1 \mathrm{~b}$ show the location of these states on a radio/Xray plot. Below is a description of each state as well as what X-ray/HXR observations have told us about each of them.

Quiescent State: It is a time of moderate radio brightness $(\sim 100 \mathrm{mJy})$, low variability and the HXR flux anti-correlates with the radio. Both the radio and X-ray fluxes vary in a correlated manner below the transition level.

A recent study [14] of the low/hard state of Cygnus X-3 using INTEGRAL found: (1) the HXR spectrum has a cutoff at $\sim 20 \mathrm{keV}$ (for most black hole XRBs in a state similar to this one the cutoff occurs at $\sim 100 \mathrm{keV}$ ); (2) there is a significant contribution from non-thermal Comptonization, usually this is observed only in soft states; and (3) the luminosity of the hard state is significantly higher than other XRBs in this state, implying a model much more radiatively efficient than the standard ADAF models or a mass of the compact object $\gtrsim 20 \mathrm{M}_{\odot}$.

Minor Flaring State: The X-ray flux oscillates around its transition value while the radio flux varies up to its transition value. The radio flux may occasionally go above its transition level, but only when the X-ray flux is above it. Overall variability in both the X-ray and radio increases. The first INTEGRAL spectrum of Cygnus X-3 [15]] was in this state.

Suppressed State: The radio flux is below its transition value and the X-ray flux is above its transition value. This state will not result in a radio flare unless the radio flux declines to the 
quenched state.

Quenched State: It is during this state that radio emission goes very low $(10-20 \mathrm{mJy})$, the HXR vanishes and the HXR flux switches from an anti-correlation to a correlation with the radio. The radio flux is well below the transition level and the X-ray flux is above it, and the state is followed by a major radio flare.

The INTEGRAL observation in May/June of 2007 is likely representative of this type of state [16], during which the X-ray spectrum was very soft and the presence of a very weak and hard $(\Gamma \simeq 1.7)$ power law tail was observed.

Major Flaring: It is during this state that the radio emission goes very high (5-20 Jy). The HXR correlates with the radio and rises. The X-ray flux is above the transition level, and the radio flux moves up and down by a large factor in a flare. A more detail discussion of the HXR behavior relative to the radio follows in this paper.

Post-Flare: This state represents the return after a major flare to either the minor flaring sta te or the suppressed one. This state is not strictly a new state since it is dominated (in the radio) by the decaying major radio flare.

\section{Hardness-Intensity Relationships}

An important tool into understanding the nature of the transient black hole (BH) systems has been hardness-intensity plots [17]. So it should be natural to look at Cygnus X-3's hardnessintensity plot and compare it to other BH systems.

\subsection{Black Hole Hardness-Intensity Plots}

A typical BH hardness-intensity plot has Q type shape [17]. It is composed of four main areas or states:

Quiescent (lower right): The source flux is very low in both the X-ray and radio with the spectrum described by a hard power law; LS (right side): The source can be bright in the X-rays and its spectrum is described by a power law with exponential cutoff at $\sim 100 \mathrm{keV}$. The radio emission has a flat spectrum and appears as a compact jet; VHS/IS (top): During this state there is a prominent thermal disk component and a steep power law without a cutoff; HS (left side): This state is thermally dominated and typically there is no power law component or if present very weak.

There is also a line that demarcates the VHS/IS region from the HS region that is referred to as the Jet Line. When the source crosses this line going from right to left there is a sudden cut off of radio emission. It is during this transition that strong radio flares occurs.

\subsection{Cygnus X-3's Hardness-Intensity Plot}

In Fig. 2 a hardness-intensity plot is shown for Cygnus X-3. The bands were chosen to be typical of those used for other BH systems. At first glance the plot appears very similar to BH hardness-intensity plot. But there are some very important differences:

Quiescent Region: Cygnus X-3 is very bright in both the X-ray and the radio (60-200 mJy) compared to other $\mathrm{BH}$ systems in this state. The spectrum shows strong iron lines and turns over at $20 \mathrm{keV}$ (not at $\sim 100 \mathrm{keV}$ as seen in other BH systems). 
Solor-intensity diagram 3-6 keV vs. $10-15 \mathrm{keV}$ of all PCA Obs.

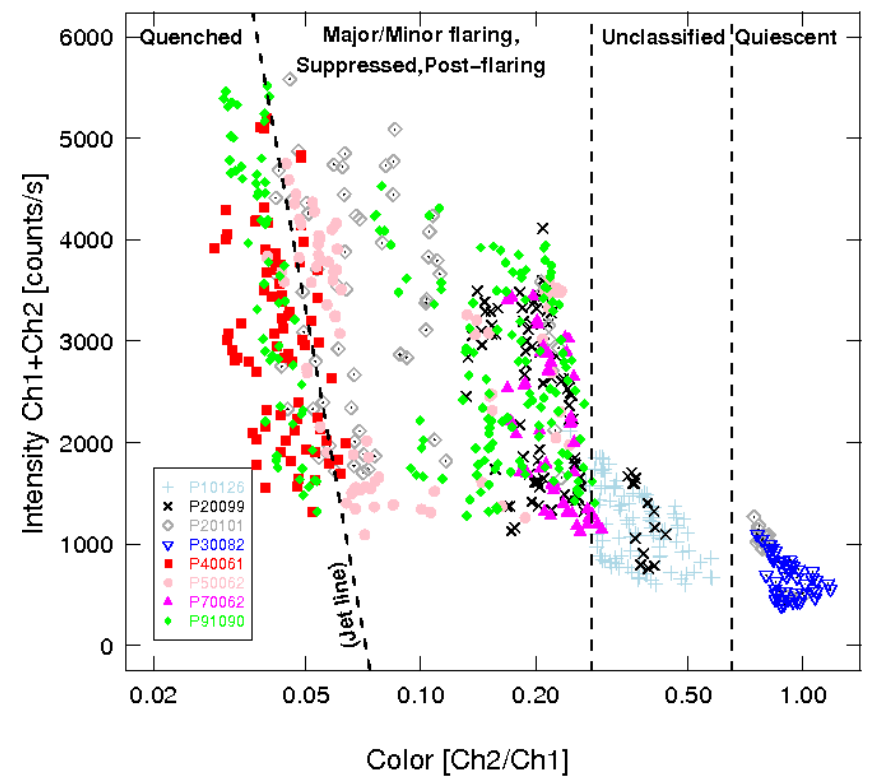

Figure 2: A hardness-intensity plot for Cygnus X-3. Note the similarity in appearance to the hardnessintensity plot for a black hole transient systems [17]. See the text for the noted differences.

Transition Region: This is where Cygnus X-3 starts exhibit minor flaring in the radio. The power law component of the spectrum starts to flatten. In Fig. 2 this region is labeled as unclassified.

Major Radio Flares (HXR): The major radio flares in Cygnus X-3, which exhibit HXR emission, are located in the left vertical branch. In a normal hardness-intensity plot for a $\mathrm{BH}$ system this would correspond to the $\mathbf{L S}$ state. The spectrum is dominated by a thermal component with a power law with no cutoff.

"Jet Line": The left branch of the hardness-intensity plot is split by a "jet line". To the left side of this line is the typical thermally dominated HS (radio/HXR quenched state). To the right of this line is a region where major radio flares occur without prominent HXR emission. VLBI observations during one these types of flares did not reveal any jet-like structure [11]. These events possibly represent some type of "failed" jet. The spectrum is again dominated by a thermal component with a weak power law. Also most remarkably Cygnus X-3 crosses the "jet line" from right to left (from minor flaring to quenching) without producing a major radio flare. It is only when it crosses from left to right that a major flare occurs.

\section{2006 Cygnus X-3 Campaign}

To better understand the nature of the major radio flares in Cygnus X-3 a multi-wavelength observing campaign was organized centered around INTEGRAL TOO observations. These were all triggered by a transition, by Cygnus X-3, into an active state in 2006. 
The observations consisted of: Chandra/HETG: Grating observations designed to probe the wind during a quenched state observation (HS); INTEGRAL: Observations to study the HXR/ $\gamma-$ ray emission of major radio flares and their decay. In addition to searching for possible annihilation lines; RXTE: Supporting pointed observations for Chandra and INTEGRAL providing both higher time resolution measurements and cross calibration. Also included were the RXTE/ASM measurements; Swift: Supporting observations for INTEGRAL, in particular for the soft X-ray coverage. These also provide additional hard X-ray and Optical/UV coverage. Ryle: High time resolution radio observations $(15 \mathrm{GHz})$; RATAN-600: Daily multi-frequency radio observations for greater spectral resolution in the radio; and PAIRITEL: Near IR ( $\mathrm{H}, \mathrm{J}$, and $\mathrm{K}$ band) observations for at least one of the observations.

\section{Cygnus X-3 INTEGRAL Observations}

During the 2006 campaign three INTEGRAL TOO observations of Cygnus X-3 ( 100 ksec duration) were planned and executed. During this campaign there were two TOO observations of EXO 2030+375 ( $200 \mathrm{ksec}$ duration) in which Cygnus X-3 is also in the field and for which the data was made available.

During this campaign there were two major radio flares (13.8 Jy at $15 \mathrm{GHz}$ and $13.2 \mathrm{Jy}$ at 2.3 GHz respectively) observed with INTEGRAL. They both fell on the right hand side of the hardnessintensity plot (HXR present). Thus we treated them as being similar in terms of how the HXRs evolved. Doing this gave a range of observations that went from within six hours of the peak up to 24.4 days after a major flare. We refer to the INTEGRAL observations according to the time from the peak of the flare with \# 1 being 6 hrs from the peak and \# 5 being 24.4 days from the peak.

For all observations simultaneous fits to the INTEGRAL/JEM-X, INTEGRAL/SGRI, and INTEGRAL/SPI data were performed. For observations for which we had Swift/XRT data we also used these data to constrain the absorption and disk temperatures. In cases where we had radio and/or near IR data these were also used in the fits.

During these observations Cygnus X-3 was a strong X-ray source but soft when compared to INTEGRAL quiescent state observations. The strong $4.8 \mathrm{hr}$ orbital modulation was seen in all of the observations.

\subsection{Cygnus X-3 Campaign (1st Flare)}

During the first major radio flare the INTEGRAL observation \# 2 took place within 2.5 days of the peak in the radio and observation \# 3 took place 6.9 days after the radio peak. The RXTE/ASM hardness ratio $[(5-12 \mathrm{keV}) /(3-5 \mathrm{keV})]$ shows an almost intermediate response to the radio flare (see Fig. 3a). But the HXR emission observed by Swift/BAT (15-50 keV) shows a three day lag.

\subsection{Cygnus X-3 Campaign (2nd Flare)}

For the second major flare observation \# 1 was within $6 \mathrm{hrs}$ of the peak. The RXTE/ASM hardness ratio $[(5-12 \mathrm{keV}) /(3-5 \mathrm{keV})]$ again shows an almost immediate response to the flare (possibly earlier). Whereas the HXR emission observed by Swift/BAT (15-50 keV) shows at least a one day lag (lower bound due to HXR sampling). See Fig. $3 b$. 

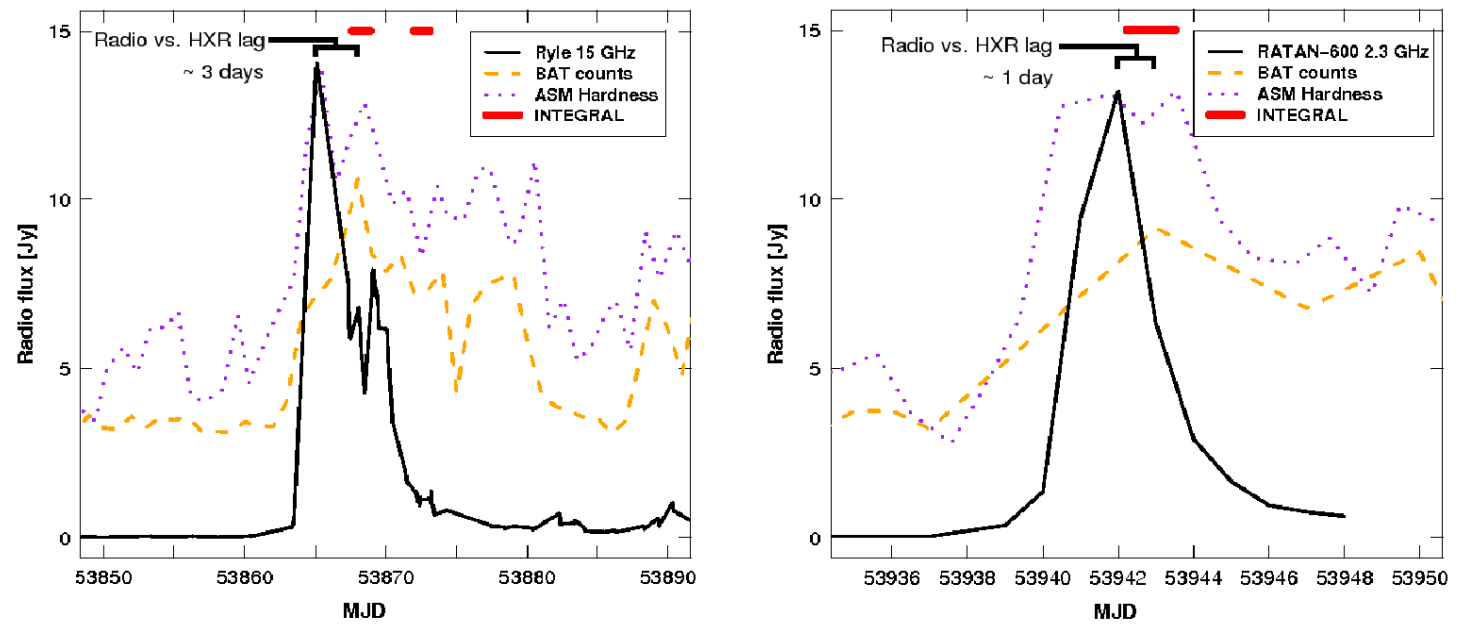

Figure 3: left (a): First major flare showing the radio (Ryle), Swift/BAT hard X-ray, and RXTE/ASM hardness ratio. Also shown are the times of the INTEGRAL observations. right $(b)$ : Second major flare showing the radio (RATAN-600), Swift/BAT hard X-ray, and RXTE/ASM hardness ratio. Also shown are the times of the INTEGRAL observations.

\section{Hard X-Ray Spectra and Time Evolution}

As a first step we perform simple fits to the HXR data provided by INTEGRAL/ISGRI. A simple power law fit is made to the $20-200 \mathrm{keV}$ spectrum for each of the observations. All fits yielded statistically satisfactory fits to the data. This was then repeated on the individual science windows ( $\sim 2 \mathrm{ksec})$ of each of the observations to examine spectral variation on a shorter timescale within each individual INTEGRAL observation.

\subsection{Spectra and Evolution Between Observations}

The HXR flux observed by INTEGRAL peaks $\sim 3$ days after the peak in the radio in agreement with what is observed in the Swift/BAT data (see Fig. 4a). The HXR spectrum starts very steep ( $\Gamma \sim 3.6)$ and as the radio flare fades the HXR spectrum becomes progressively harder $(\Gamma \sim 2.3)$. It appears that as the major radio flare fades there may be ongoing processes that are effecting the particle distribution which is associated with the production of the HXRs.

\subsection{Short Timescale Evolution (science window)}

To examine spectral changes on a shorter timescale we performed power law fits for each of the science window of each observation. Individual science windows at the beginning and at the end of the observations were dropped. Also science windows impacted by high background levels were dropped out of the analysis.

Observations 1-5 are in time order from the peak of the most recent major radio flare (see Fig. $4 \mathrm{~b}$ and $4 \mathrm{c}$ ). In observations 1-2, and 4 there appears little spectral evolution occurring during the INTEGRAL observation. Observation 3 shows an abrupt hardening of the spectra during about a 

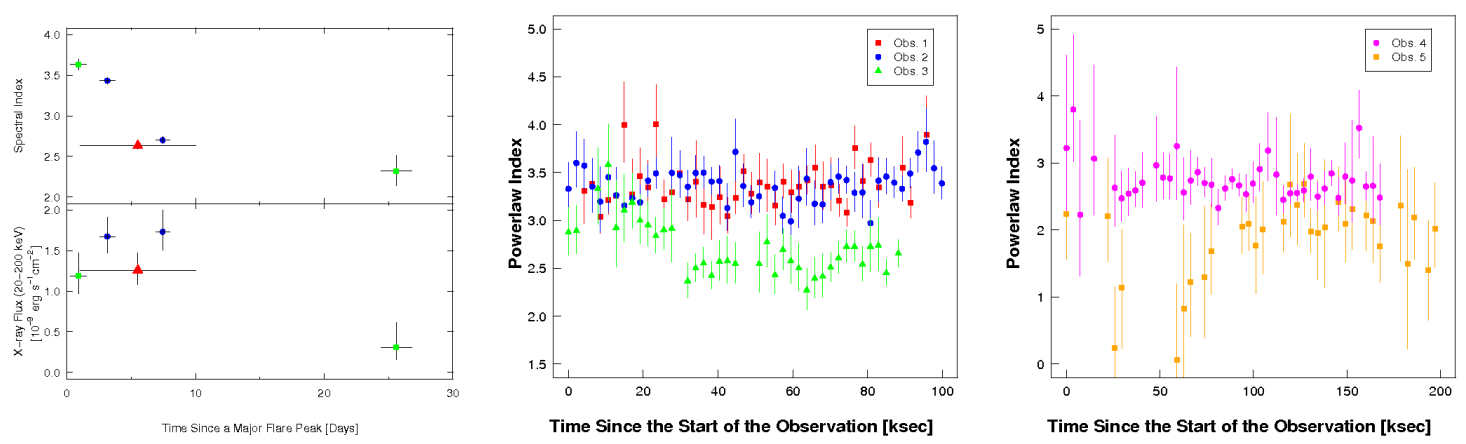

Figure 4: left (a): Plot of the power law index, from a fit to the 20-200 keV INTEGRAL/ISGRI spectrum, as a function of time from the peak of a major radio flare. center $(b)$ : A plot of the power law index,from a fit to the 20-200 keV INTEGRAL/ISGRI spectrum, for each science window of observations 1, 2, and 3 . right (c): A plot of the power law index, from a fit to the 20-200 keV INTEGRAL/ISGRI spectrum, for each science window of observations 4 and 5 .

quarter of the observation after which it remains relatively constant. Observation 5 may indicate some hardening with time.

The spectral evolution appears to be slow, on timescales of days. But there are indications that there may be short abrupt hardening of the spectra. This may indicate that the HXR spectra is evolving in a stochastic manner. This may be what is expected in internal shocks within the jet which are giving rise to particle acceleration and modifying the particle distribution.

\section{Detailed Spectral Model}

To better understand the INTEGRAL spectra each of the observations were fit with a more complex model. The main component of our fits was hybrid plasma Comptonization model (eqpair [18]). In addition to the eqpair model we also used: (a) Absorption (phabs); (b) Partial covering fraction absorption (pcfabs); (c) Disk Blackbody (diskbb); (d) Absorption edge (edge); (e) Emission lines (gauss) of $\mathrm{Fe}, \mathrm{Si}$, and $\mathrm{S}$; and (f) For observations which we had radio and near IR we also included a synchrotron model (sync) and reddening (redden) to do a joint fit to the data. Also when available we used Swift/XRT data to constraint the absorption and disk temperature.

In the following discussion we will concentrate on the X-ray/HXR region of the spectrum. In all cases presented here a power law form of the nonthermal particle injection was used. For a fuller discussion of the fitting of complete multi-wavelength data sets see [19].

\subsection{INTEGRAL Spectra}

The results of the fits are shown in Fig. 5 and some of the various fit parameters/results are contained in Tables 1-3. For all of the observations a good consistent fits to the data was found. In Table 1 are fit parameters that showed a consistent range of values for all observations, Table 2 has select eqpair fit parameters, and Table 3 has the flux estimates and the breakdown by major spectra components. There are several points to note: 


\begin{tabular}{|c|c|c|}
\hline Absorption: & $\mathrm{N}_{\mathrm{H}, 0}$ & $2.37-3.56 \times 10^{22} \mathrm{~cm}^{-2}$ \\
\hline Partial Covering: & $\mathrm{N}_{\mathrm{H}, 1}$ & $0.92-1.17 \times 10^{23} \mathrm{~cm}^{-2}$ \\
\hline Partial Covering: & $\mathrm{f}_{\mathrm{c}}$ & $0.81-0.85$ \\
\hline Edge: & $\mathrm{E}_{\mathrm{e}}$ & $9.28 \mathrm{keV}(\mathrm{fixed})$ \\
\hline Reddening: & $\mathrm{E}(\mathrm{B}-\mathrm{V})$ & $\sim 5.0$ \\
\hline Disk Temperature: & $\mathrm{kT}_{\mathrm{bb}}$ & $0.76-1.32 \mathrm{keV}$ \\
\hline
\end{tabular}

Table 1: Table of non-Eqpair fit parameters which have a limited range of values in the fitting.

\begin{tabular}{|c|c|c|c|c|c|}
\hline Time & 0.25 days & 2.51 days & 6.88 days & $1-10$ days & 24.4 days \\
\hline \hline Eqpair (power law): & & & & & \\
\hline $1_{\mathrm{nt}} / \mathrm{l}_{\mathrm{h}}$ & $0.62-1.00$ & $0.29-1.00$ & $0.83-1.00$ & $0.78-1.00$ & $0.70-1.00$ \\
\hline $\mathrm{l}_{\mathrm{h}} / \mathrm{l}_{\mathrm{s}}$ & 0.88 & 0.66 & 0.34 & 0.28 & 0.35 \\
\hline$\tau$ & 3.83 & 3.93 & 1.33 & 1.87 & 9.41 \\
\hline$\Gamma$ & 2.36 & 4.17 & 3.69 & 3.33 & 1.82 \\
\hline \hline$\chi_{v}^{2} /$ d.o.f. & $1.12 / 193$ & $1.60 / 174$ & $1.01 / 190$ & $1.48 / 83$ & $1.07 / 82$ \\
\hline
\end{tabular}

Table 2: Table of select Eqpair + Synchrotron fit parameters and their fit values.

Nonthermal Component: In order to fit the data it was necessary for there to be a major nonthermal component in the plasma providing the Comptonized emission. This is in keeping with on-going jet activity.

Absorption: The absorption is found to be around $3 \times 10^{22} \mathrm{~cm}^{-2}$ for all of the observations. This is consistent with the observed $\mathrm{HI}$ observations allowing for extra absorption due to molecular clouds along the line of sight. There is also need for partial covering factor with a value of $\sim$ $10^{23} \mathrm{~cm}^{-2}$. This is in keeping with strong wind and accretion disk that are likely associated with Cygnus X-3.

Emission Lines: To achieve reasonable fits the three brightest emission lines needed to be fit separately. In terms of total flux they change very little between observations.

Fe Edge: All the observations consistently show an edge at $\sim 9 \mathrm{keV}$. This edge would naturally occur from the presence of highly ionized $\mathrm{Fe}$ which is know to exist from the emission line spectrum [20]. Since the edge was not prominent but improves the fits its value was frozen at 9.28 $\mathrm{keV}$.

Luminosity: As can be seen from Table 3 Cygnus X-3's luminosity is quite high throughout these observations. For a $10 \mathrm{M}_{\odot}$ compact object this would correspond to $0.12-0.21 \mathrm{~L}_{\mathrm{Edd}}$. It is also relatively constant. If radio or near IR observations were available for the last two observations it is likely the flux variation between observations would even be less.

Jet Luminosity: It is also notable that the jets in Cygnus X-3 contribute a large amount to the overall luminosity of the system (up to $27 \%$ ). In all likelihood they play a major role in the overall dynamics of the system and how Cygnus X-3 interacts with the environment. 


\begin{tabular}{|c|c|c|c|c|c|}
\hline Time & 0.25 days & 2.51 days & 6.88 days & $1-10$ days & 24.4 days \\
\hline \hline Absorbed: & & & & & \\
\hline $\mathrm{L}_{\text {tot }}\left(10^{38}\right.$ ergs $\left./ \mathrm{sec}\right):$ & 0.94 & 0.99 & 1.22 & 0.74 & 0.81 \\
\hline $\mathrm{F}_{\text {sync }} / \mathrm{F}_{\text {tot }}$ & 0.04 & 0.03 & 0.02 & - & - \\
\hline $\mathrm{F}_{\text {disk }} / \mathrm{F}_{\text {tot }}$ & 0.29 & 0.04 & 0.00 & 0.00 & 0.20 \\
\hline $\mathrm{F}_{\text {comp }} / \mathrm{F}_{\text {tot }}$ & 0.65 & 0.90 & 0.95 & 0.98 & 0.79 \\
\hline $\mathrm{F}_{\text {lines }} / \mathrm{F}_{\text {tot }}$ & 0.02 & 0.03 & 0.03 & 0.02 & 0.01 \\
\hline \hline Unabsorbed: & & & & & \\
\hline $\mathrm{L}_{\text {tot }}\left(10^{38}\right.$ ergs $/$ sec $):$ & 2.62 & 2.90 & 2.90 & 1.58 & 2.55 \\
\hline $\mathrm{F}_{\text {sync }} / \mathrm{F}_{\text {tot }}$ & 0.27 & 0.25 & 0.15 & - & - \\
\hline $\mathrm{F}_{\text {disk }} / \mathrm{F}_{\text {tot }}$ & 0.40 & 0.16 & 0.00 & 0.00 & 0.41 \\
\hline $\mathrm{F}_{\text {comp }} / \mathrm{F}_{\text {tot }}$ & 0.31 & 0.56 & 0.82 & 0.99 & 0.58 \\
\hline $\mathrm{F}_{\text {lines }} / \mathrm{F}_{\text {tot }}$ & 0.02 & 0.03 & 0.03 & 0.01 & 0.01 \\
\hline
\end{tabular}

Table 3: Table of absorbed/unabsorbed luminosities and contribution of the various components. To determine the luminosities a distance of $9 \mathrm{kpc}$ was assumed for Cygnus X-3

\subsection{Evolution of the INTEGRAL Spectra}

The sequence of INTEGRAL observations give us a unique view of how the X-ray/HXR spectrum evolves as a major radio progresses. Among the things to note:

High Energy Spectrum: While the overall flux remains relatively constant for these observations, the flux is shifting from high energy spectrum to the softer part of the spectrum as it evolves. Throughout the evolution the high energy spectrum tends to be dominated by the nonthermal part of the particle spectrum.

Particle Injection Index: The first observation shows that particle acceleration is important for the initial observation. The next observation exhibits a very steep distribution. This would indicate particle acceleration has become less efficient. One possible interpretation would be particles created by magnetic reconnection being ejected from the disk through the jet. In progressive observations we see the index flattens. Until in the last observation it appears that some type of particle acceleration has become more efficient.

Optical Depth: The initial optical depths indicate that the nonthermal particles radiate before having a change to thermalize. As the flare progresses the overall optical depth starts to drop. But by the last observation the environment near the compact object has changed and the optical depth is now quite large. This is also one of the reasons why we see the very hard nonthermal tail in the last observation.

Synchrotron: For the observations for which there are matching radio observations there is direct evidence that the jets are very important energetically for Cygnus X-3. The synchrotron appears to extend into the soft X-ray but not into the HXR. The contribution of the jet to the overall energy budget appears to decrease as the radio flare decays (as might be expected). But the current lack of radio observations for the last two limits what we can say. See [19] for a fuller description of the fitting of the overall Spectral Energy Distribution (radio to $\gamma$-ray). 

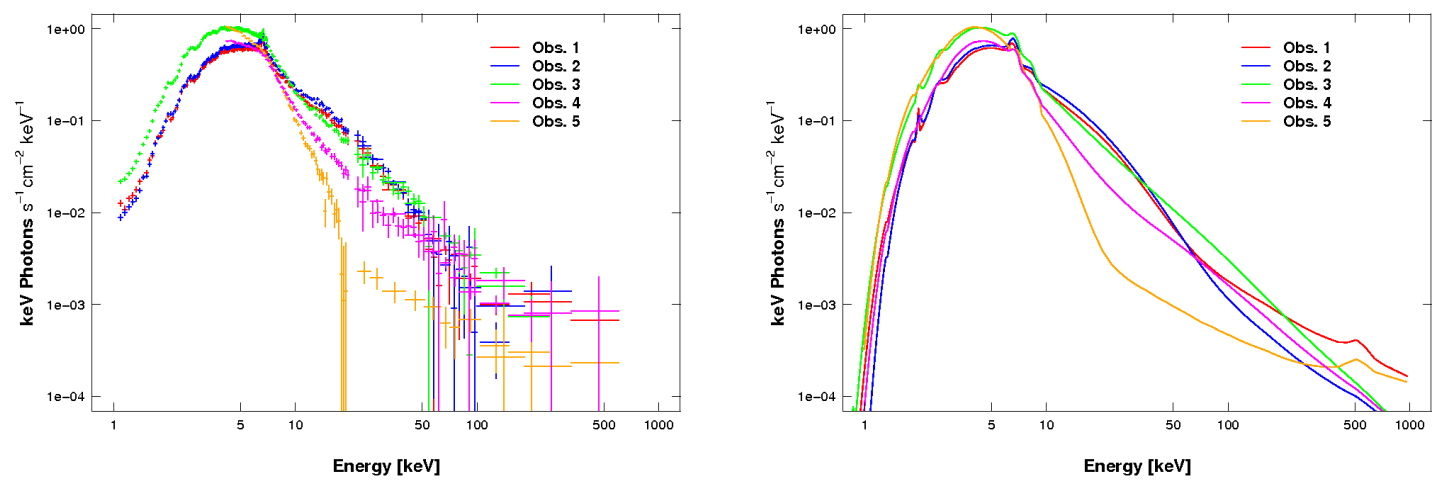

Figure 5: left:INTEGRAL data for the five Cygnus X-3 observations. right: Eqpair model fits for the Cygnus $\mathrm{X}-3$ observations.

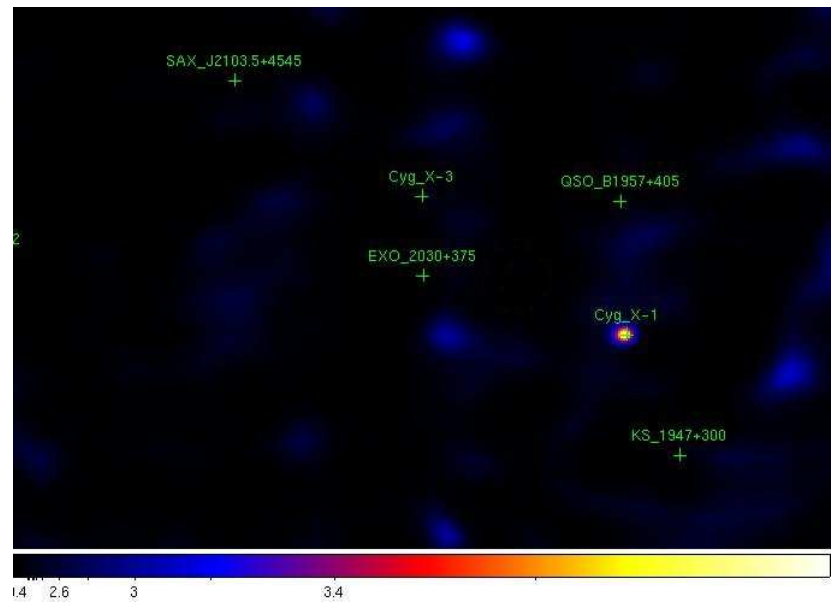

Figure 6: Image made from the INTEGRAL/SPI data centered on the $511 \mathrm{keV}$ energy with a width of 70 $\mathrm{keV}$ for observation \# 1 . No evidence of an annihilation line from Cygnus X-3 is apparent.

\section{Annihilation Lines}

For the jets produced by microquasars like Cygnus X-3 there is a question of the composition of the jets. Are the jets mostly lepton (pair plasma) or more baryonic such as those observed in SS433? Since the INTEGRAL observations are near the peak of major flares they are prime observations to search for annihilation lines.

All of the INTEGRAL/SPI observations were searched for annihilation line emission (511 $\mathrm{keV}$ ). Many models predict that the annihilation for such a jet would appear as a broad emission lines $(\sim 70 \mathrm{keV})$ [21]. We have searched all five data sets, but we haven't found evidence of such lines (see Fig. 6). For some of the observations we find some indications of narrow lines. But a better refined background modeling of the data needs to be done before we can access the reality of these lines. It is interesting that for some of eqpair fits the model hint at a possibility of annihilation line emission. 


\section{Summary}

We have provided the first INTEGRAL examination at the nature and evolution of the Xray/HXR spectrum associated with major radio flares in Cygnus X-3. Among the things we have discovered in this study are: (a) There are two types of major radio flares in Cygnus X-3. The major flares that fail to produce HXRs (or produce them at a greatly reduced rate), which may be the result of a "failed" jet, possible as the result of an interaction between the jet and the companion star; (b) HXR flares start out with a steep HXR spectrum which progressively becomes harder as the flare evolves; (c) There is a delayed response in the HXR relative to the radio. Also there are indications that the amount of delay depends upon the X-ray energies; (d) No evidence for strong annihilation emission near or past the peak of the flare have been observed; (e) Synchrotron emission provides a major energy component to the spectrum; and (f) There appears to be a "jet line" in Cygnus X-3's hardness-intensity diagram. But major radio flares are produced only when going the opposite direction in the diagram than is observed in BH transients.

These results are exciting and give rise to a whole set of new questions about Cygnus X-3 and its nature. This analysis is continuing.

\section{References}

[1] van Kerkwijk, M. H. et al. 1992, Nature, 355, 703

[2] Molnar, L. A. et al. 1988, ApJ, 331, 494

[3] Schalinski, C. J. et al. 1995, ApJ , $\mathbf{4 4 7}, 752$

[4] Mioduszewski, A. J. et al. 2001, ApJ, 553, 766

[5] Waltman, E. B. et al. 1996, AJ, 112 , 2690

[6] McCollough, M. L., et al., 1999, ApJ, 517, 951

[7] Szostek, A., Zdziarski, A. A. \& McCollough, M. L. ,MNRAS, 388, 1001

[8] McCollough, M. L. et al. 1997, in "Transparent Universe”, ESA, SP-382, 265

[9] McCollough, M. L. et al. 1997, 4th Compton Symposium, 813

[10] McCollough, M. L. et al. 1998, New Astr. Rev., 42, 629

[11] McCollough, M. L. et al. 1999, BAAS, 31, 742

[12] McCollough, M. L. et al. 1999, Astro. Lett. and Comm., 38, 105

[13] Watanabe, H. et al. 1994, ApJ, 433, 350

[14] Hjalmarsdotter L. , et al. 2008, MNRAS, 384, 278

[15] Vilhu, O., et al. 2003, A\&A, 411, L405

[16] Beckmann, V., et al. 2007, $A \& A, \mathbf{4 7 3}, 903$

[17] Fender, R. P., Gallo E., \& Belloni, T. E., 2004 MNRAS, 355, 1105

[18] Coppi, P. S., 1992 MNRAS, 258, 657

[19] Koljonen, K. I. I. \& McCollough, M. L., 2008, these proceedings

[20] Verner, D. A. et al. 1996, ApJ, 465, 487

[21] Ramaty, R. \& Meszaros, P. 1981, ApJ, 250, 384 